Shear Wave Propagation in Granular Assemblies

\author{
Z. Ning ${ }^{1}$, A. Khoubani ${ }^{2}$, and T.M. Evans ${ }^{3}$
}

\begin{abstract}
Shear wave velocity is a fundamental property of a granular assembly. It is a measure of the true elastic stiffness of a bulk specimen of discrete grains. Shear wave velocity is typically measured in the laboratory (e.g., using bender elements) or in-situ (e.g., using a seismic cone penetrometer, sCPT). In the current work, shear wave propagation is modeled numerically using the discrete element method (DEM). First, an appropriate method for measuring wave velocity is identified. The effects of particle size and elastic properties are investigated. Specimen fabric is quantified before and after wave excitation and the elasticity of the response at the scale of the particle contacts is investigated. The results show that shear wave velocity may be robustly measured for discrete numerical specimens. The ability to measure shear wave velocity using DEM simulations may provide another tool for researchers seeking to link results from physical and numerical experiments.
\end{abstract}

\title{
INTRODUCTION
}

The small strain shear modulus $G_{\max }$ is widely recognized as a fundamental soil stiffness property important for design and analysis of various geotechnical structures (Burland 1989; Patel et al. 2009; Clayton 2011). It is a critical input for multiple constitutive models used to describe the static and dynamic behavior of granular materials (e.g., Santamarina et al. 2001). In practice, $G_{\max }$ is normally indirectly obtained by measuring shear (S-) wave velocity $V_{s}$, which is related to $G_{\max }$ by the well-known formula:

$$
G_{\max }=\rho \cdot V_{s}^{2}
$$

where $\rho$ is the soil bulk density (e.g., Santamarina et al., 2001).

The laboratory techniques now available to measure S-wave velocity of soils are quite diverse, such as bender element tests (Shirley and Hampton, 1978; Viggiani and Atkinson 1995; Lee and Santamarina 2005) and resonant column tests (Hardin and Richart 1963). There are also in-situ tests to measure $V_{s}$ such as seismic cone penetration test (sCPT; Robertson et al. 1995). Among those, bender element test is the most prevailing method due to its usefulness and simplicity. A bender element is a piezoelectric transducer that can convert mechanical distortion to voltage change and vice-versa due to the phenomenon of piezoelectricity. A typical bender element test system consists of a transmitter and a

\footnotetext{
1 Project Engineer, SOLDATA Northern America, 1448 Elliott Ave. W., Seattle, WA 98119

${ }^{2}$ Graduate Research Assistant and Jerry Yamamuro Fellow, School of Civil and Construction Engineering, Oregon State University, 101 Kearney Hall, Corvallis, OR 97331

${ }^{3}$ Associate Professor, School of Civil and Construction Engineering, Oregon State University, 101 Kearney Hall, Corvallis, OR 97331, Corresponding author: 541.737.8535, matt.evans@oregonstate.edu
} 
receiver, anchored to the top or the bottom platens of standard static testing devices (e.g. triaxial apparatus and oedometer) respectively. When a voltage is applied to the transmitter, shear motions will be generated and propagate through the soil and detected by the receiver. The maximum shear strain induced by bender element was estimated by Dyvik and Madshus (1985) to be less than $10^{-5}$. Then $V_{s}$ is simply calculated as the distance between the transmitter and the receiver divided by the travel time. There is increasing interests in using S-wave velocity to define particle parameters (e.g. shape, elastic properties, gradation; Patel et al. 2009; Yang and Gu 2012) and state (e.g. void ratio, boundary stress; Hardin and Richart 1963). Another large body of research focuses on using the small strain modulus $G_{\max }$, calculated from S-wave velocity $V_{s}$ to predict the large-strain soils parameters (e.g. undrained/drained shear strength; Saxena et al. 1988; Sharma et al., 2011). For all these S-wave related studies where bender element tests are used, accurate determination of S-wave travel time has long been a critical but controversial topic. However, due to the inherent complex particle motions, interfering side effects (e.g. cross-talk; compression (P-) wave interference; Lee and Santamarina 2005) and fabrication deficiencies (Montoya et al. 2013), the measurement uncertainty can lead to an error of $30-50 \%$ (Viggiani and Atkinson 1995; Arroyo 2003).

Discrete element method (DEM) simulation is a useful tool for investigating the complex behavior of particulate materials in conjunction with laboratory tests. In terms of wave propagation, twodimensional DEM simulations have been conducted to study the general relationships between wave propagation variables and soil fabric (Sadd et al. 1993). Li and Holt (2002) investigated the acoustic properties of weakly cemented sandstone based on elastic wave propagation using three-dimensional DEM simulations. O'Donovan et al. (2012) recently used a two-dimensional DEM model with ideal hexagonally-packed monodisperse discs to simulate bender element tests. This prior work demonstrates the ability of DEM simulations to capture the parameters of interest which are difficult or very expensive to directly observe in standard physical tests.

The object of this work is to study both specimen-scale and particle-scale responses of threedimensional granular assemblies during S-wave propagation in an environment which is similar to the physical bender element test, so as to provide insights for the design of physical experiments and potentially a more robust linkage between physical and numerical experiments. The simulation of DEM specimen, S-wave generation and measurement are presented first, followed by an examination of system response at the particle level. Receiving signals from multiple receivers were interpreted with different methods. Finally the effects of excitation signals, particle properties and stress state on S-wave velocity are analyzed.

\section{Specimen Generation}

Shear wave propagation was simulated using the DEM program $P F C^{3 D}$ (Itasca 2009). Cylindrical DEM specimens having a diameter $(\mathrm{D})$ to height $(\mathrm{H})$ ratio of 3:2 were modeled. The high $\mathrm{D} / \mathrm{H}$ ratio was employed to minimize boundary effects and compression ( $\mathrm{P}-$ ) wave reflection observed in assemblies having typical (i.e., $\mathrm{D} / \mathrm{H}=0.5$ ) triaxial specimen geometry. Particles were simulated as spheres with 
71 walls defined the top and bottom boundaries of the specimen and were used to control the applied 72 vertical stress. Radial confinement was supplied by stacked cylindrical walls to simulate a flexible 73 membrane (Zhao and Evans, 2009). The basic DEM model properties and S-wave measurement 74 parameters are listed in Table 1 . Some of these properties were subsequently varied as part of a 75 parametric study. In order to increase the allowable solution time increment, mass scaling of 1000:1 was 76 used. In this type of scaling, particle sizes are increased while particle densities remain constant. It has

77 been shown that mass-scaled DEM simulations reasonably mimic microscale evolution in real granular 78 materials (Evans and Frost 2007; Belheine et al., 2009; Evans and Frost, 2010; Evans and Valdes, 2011).

79 Simulations were conducted in the absence of gravity to prevent body force-induced stress gradients 80 from arising due to the scaled particle sizes.

81 Table 1. Basic DEM model properties and S-wave measurement parameters

\begin{tabular}{ll}
\hline Parameters & Value \\
\hline Particle properties & Hertz-Mindlin \\
Contact Model & 2.65 \\
Specific gravity, $G_{s}$ & $2.9 \times 10^{9} \mathrm{~Pa}$ \\
Particle shear modulus, $G_{g}$ & 0.31 \\
Particle Poisson's ratio, $v_{g}$ & 0.31 \\
Interparticle friction coefficient, $\mu$ & $0.205 \mathrm{~m}$ \\
Mean particle diameter, $d_{50}$ & 1.2 \\
Uniformity coefficient, $C_{u}$ & \\
Specimen geometry & 43.9 \\
Specimen diameter $/ d_{50}$ & 29.3 \\
Specimen height/ $d_{50}$ & \\
S-wave measurement parameters & $150 \mathrm{kPa}$ \\
Confining stress & $5 \times 10^{-5}$ \\
Excitation amplitude $/ d_{50}$ & $50 \mathrm{~Hz}$ \\
Excitation frequency &
\end{tabular}


82

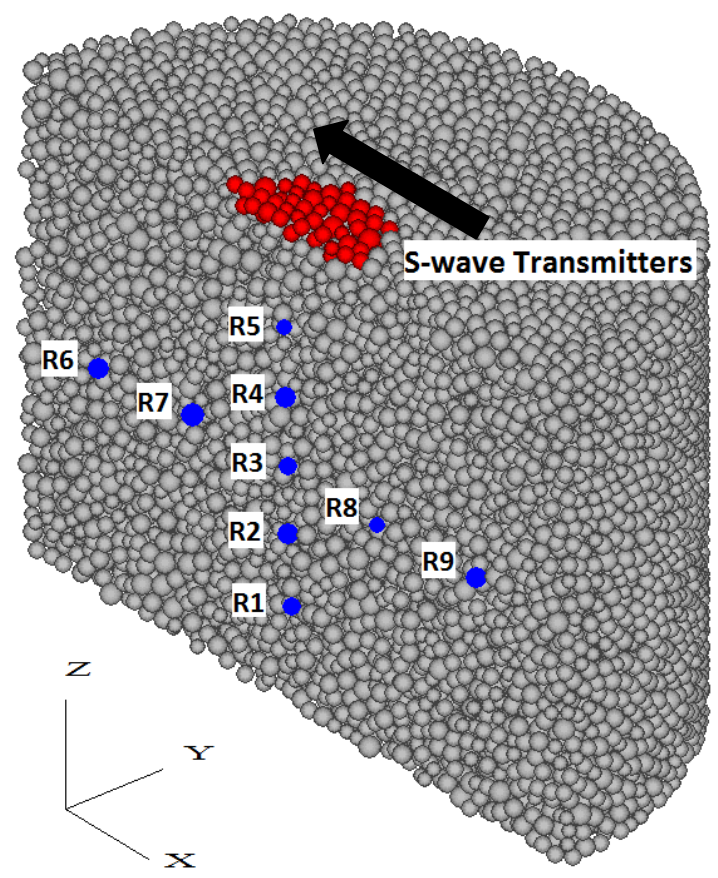

(a)

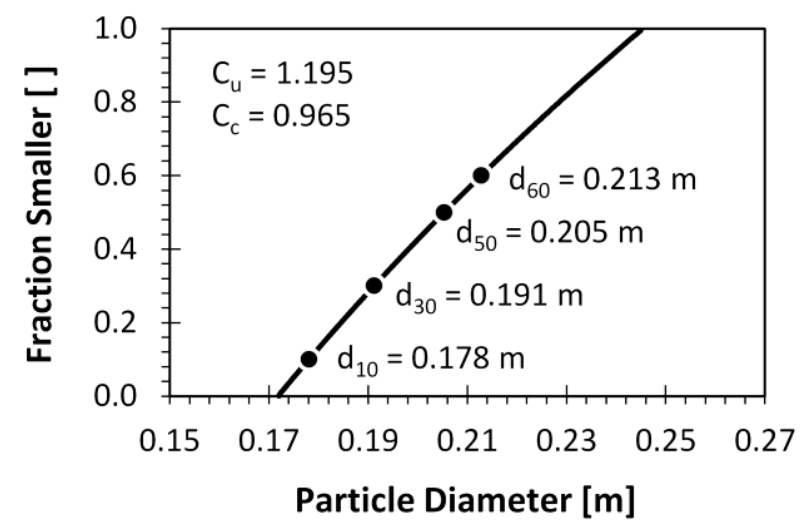

(b)

Figure 1. (a) A typical DEM specimen with S-wave transmitting zone and receivers (R1, R2, R3, R4 and R5 are primary receivers; R6, R7, R8 and R9 are complementary receivers. For clarity, only half of the specimen is shown.). (b) Grain size distribution of specimen.

\section{Shear Wave Generation and Receiving}

S-waves were generated by applying a horizontal excitation to a thin layer of particles at one end of the specimen using a single sinusoidal velocity pulse. The DEM models can be excited by sine pulses with different frequencies to approximately identify the resonant frequency (Ning and Evans, 2013). The excitation frequency was selected near the resonant frequency to obtain strong frequency response in the model. The diameter of this S-wave transmitting zone is $D / 4$ ( $D=$ specimen diameter) and its thickness is nominally $2 \times d_{50}$ (i.e., it is comprised of particles whose centroids lie within $1.5 \times d_{50}$ of the top 
of the specimen). Compared to the S-wave generation by bender elements, in which the wave source is a point and the wave propagation front is spherical, this approach reduces the P-wave interference effect (Lee and Santamarina 2005) observed in physical specimens (Ning and Evans 2013).

In a physical test, bender elements are typically installed on the boundary of a specimen (e.g., the end platens of a triaxial device or oedometer cell), which makes it difficult to access the information within the specimen - that is, the measured shear wave velocity will be a spatially averaged quantity over the height of the specimen. In DEM simulations the displacement and velocity of each individual particle is known, allowing for multiple wave receivers at specified locations to monitor particle motions during wave propagation. In the current study, five approximately equally spaced particles along the central axis of the cylindrical specimen were selected as primary S-wave receivers (R1-R5, Figure 1). In addition, four complementary receivers (R6-R9) were defined on a line orthogonal to the z-axis and parallel to the direction of shear excitation (i.e., the x-axis). Multiple receivers allow for a wider variety of procedures to interpret S-wave velocity.

\section{Simulation Process}

Simulation of shear wave propagation consists of the following: (1) specimen generation; (2) preexcitation preparation; and (3) shear wave transmission and measurement.

In step one, the DEM specimen was assembled with a predefined void ratio and isotropically consolidated to a certain stress state. In step two, the model was cycled with high local damping to ensure that pre-excitation particle motions are negligible compared to post-excitation particle motions. Once all particles in the assembly are sufficiently still, the local damping ratio is returned to a physically realistic value. Finally, a sinusoidal velocity pulse with pre-defined amplitude, frequency, and duration was applied to the particles located in the shear-wave transmitting zone. The displacement and velocity of the receivers were monitored.

Energy is dissipated in DEM simulations via frictional sliding and contact dissolution. However, because the contact laws used in the current simulation are purely elastic, there is no asperity yield at particle contacts (O'Sullivan 2011). Therefore, the amount of energy dissipated may not be sufficient to prevent non-physical vibrations in the particles (Itasca 2009). In the current work, viscous damping was used to prevent unrealistic particle motion.

\section{Signal Analysis}

Figure 2 shows the source and receiving signals in terms of the $x$ component of displacement and velocity (i.e., in the direction that the particles in the transmitting zone were excited) from a single typical S-wave propagation simulation. The signals as recorded at the receivers are designated $R 1$ to $R 5$ (from the furthest to the nearest transmitter, respectively) while $S$ denotes the source signal. Unlike most receiving signals obtained in laboratory bender element tests (e.g. Viggiani and Atkinson 1995), these signals are very 'clean' because they are free of many factors which may exist in laboratory tests such as cross-talk, electromagnetic noise, and background mechanical vibration (Lee and Santamarina 2005; Montoya et al. 2012). This high signal quality results in particularly robust determinations of travel time. Moreover, results of bender element test are in terms of displacement; however, in the numerical 
model there is access to the receivers' displacement and velocity. Both displacement and velocity signals were used for determining a more precise shear wave velocity, as explained below.

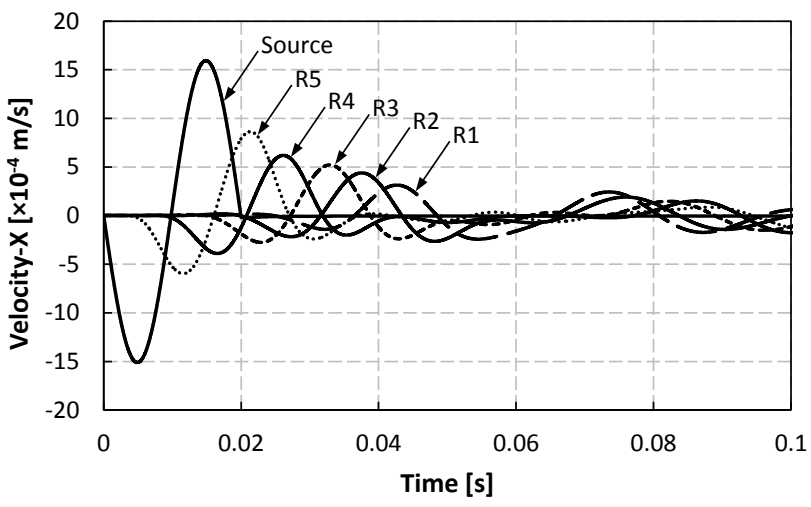

(a)

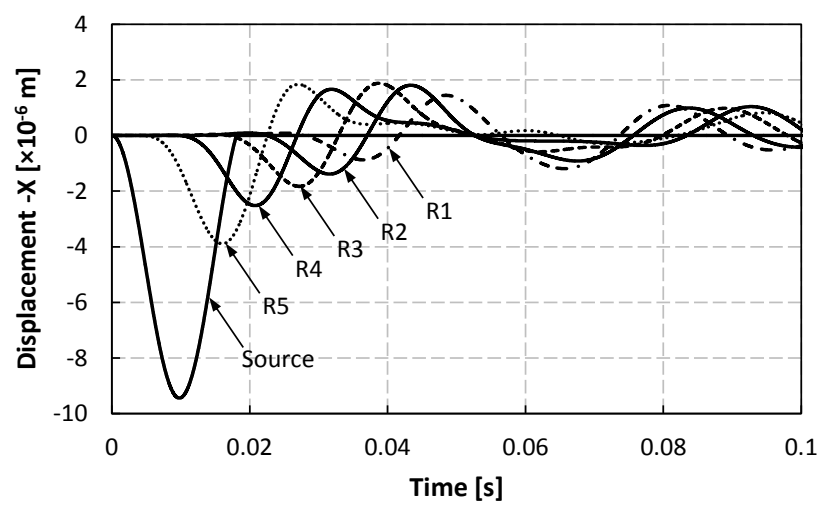

(b)

Figure 2. Transmitting and receiving signals for S-wave velocity measurement. (a) x-velocity response; (b) xdisplacement response

The source velocity signal shown in Figure 2(a) is a single-period sinusoidal pulse, which coincides with the input shear wave parameters. The source displacement signal shown in Figure 2(b) basically matches the integration of the source velocity signal over the duration of the transmitting signal, showing the particles in the transmitting zone were first moved in the $-X$ direction and then moved back to their original location. In both the velocity and displacement signals, it can be seen that the receivers at different locations respond to the excitation at different times (from the earliest to the latest: $R 5, R 4, R 3$, $R 2, R 1)$. The delays of the receiving signals between equally spaced receivers are almost the same and the receiving signals attenuate as the distance from the source increases. The receiving signals also indicate reflection at the bottom boundary of the specimen. From around 0.06-0.08 s, it can be seen that the receivers' responses - which had already been attenuated - again increased but in an inverted sequence: the closer to the base of the specimen, the earlier the response (from the earliest to the latest: $R 1, R 2, R 3, R 4, R 5)$.

\section{EXAMINATION OF SYSTEM RESPONSE}

DEM simulations allow for both particle-level and specimen-level analyses. The evolution of the particle velocity vector field and local strain contours are used to analyze the complicated system response during shear wave propagation and also to help to correctly interpret the wave receiver signals in order to determine S-wave velocity. Figure 3 shows the evolution of instantaneous particle velocity vectors during S-wave propagation at four successive time points $(10,20,30$, and $40 \mathrm{~ms}$ after excitation) on the $x-z$ and $y-z$ planes shown in Figure 1 . The particle velocity vectors are plotted in Figure 3 with their length proportional to the magnitude of velocity (the largest vector corresponds to a velocity of $3 \times 10^{-4}$ $\mathrm{m} / \mathrm{s}$ ). These four snapshots of particle velocity vectors combined with the receiving signals in time domain shown in Figure 2 demonstrate the interplay between particle-scale and specimen-scale behavior as the S-wave propagates though the specimen. 


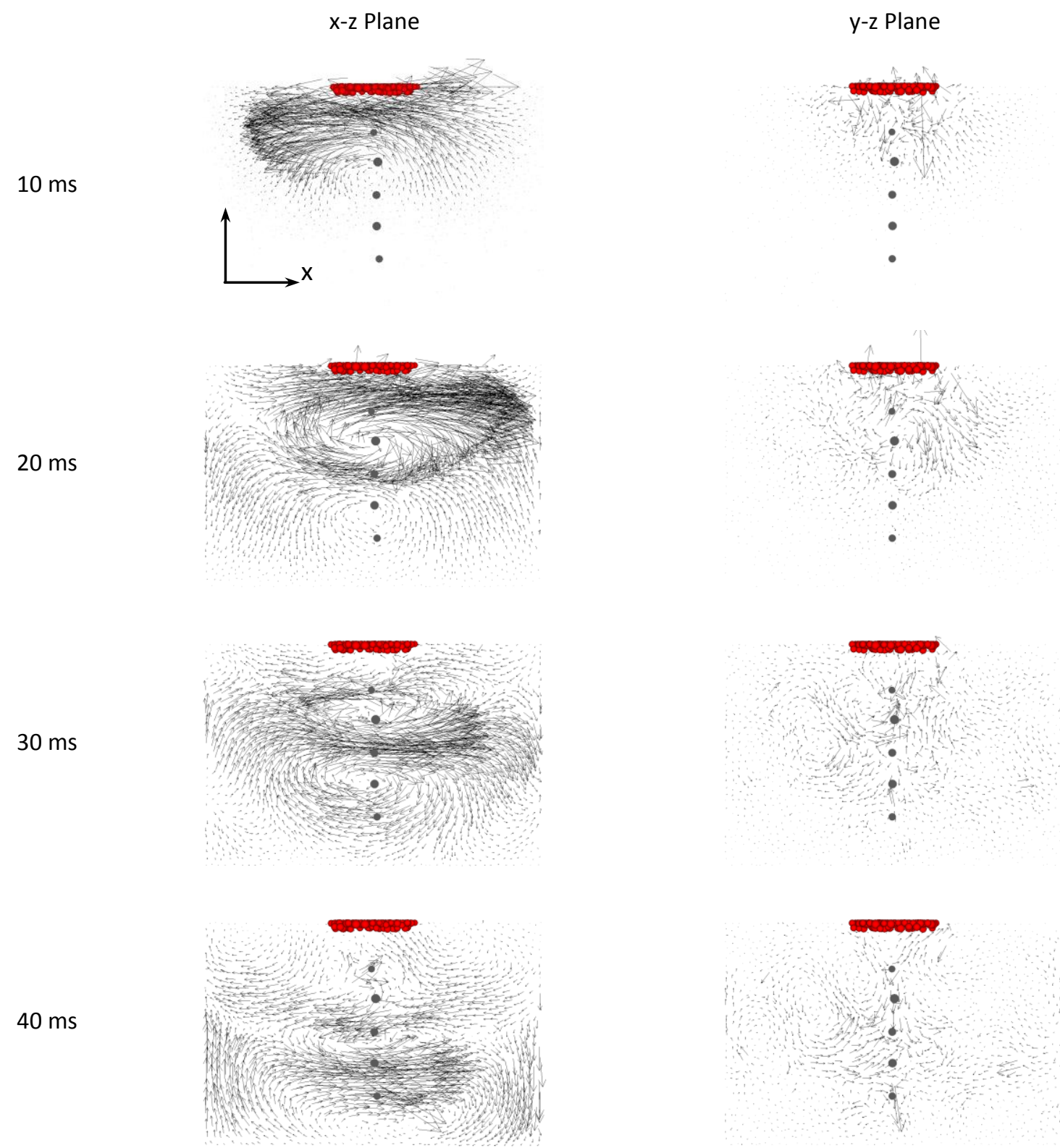

Figure 3. Instantaneous particle velocity vectors on $x-z$ and $y-z$ planes at 10, 20, 30, and $40 \mathrm{~ms}$ after excitation. Transmitter particles are shown in red while the five primary receiver particles are shown in grey. Note that all velocity arrows are plotted at the same scale.

165 At $10 \mathrm{~ms}$, as shown in Figure 2, the velocities of S-wave transmitting particles are zero and their displacements reach the peak value in the $x$-direction. Receiver $R 5$ has moved towards the $x$-direction while receiver R4 remained quiet. In Figure 3, strong horizontal particle motions in the $x$-direction are indicated by the velocity vectors in the zone between the S-wave transmitting zone and receiver $R 5$. It can also be observed that to the left of the zone in front of the shear motion, particles have a tendency to move downwards while to the right of the zone in front of shear motion, particles have a tendency to move upwards, which forms a vortex-like disturbance ahead of the main shear motions. These vortices

172 bear some resemblance to the experimental and numerical observations of others (e.g., Rechenmacher 173 2006; Tordesillas et al., 2008), but the underlying mechanics are fundamentally different. Specifically, 
Tordesillas et al. (2008) noted that the emergence of vortices (along with so-called microbands) as the predominant mode of nonaffine deformation were an indicator of impending shear band formation coinciding with peak stress ratio. With respect to wave propagation, however, there is no large-scale failure impending. Nonetheless, the observed vortices correspond with unjamming events (i.e., initiation of particle motion from an initially jammed state) and are associated with very small, local deformations, similar to observations reported by Tordesillas et al. (2008).

At $20 \mathrm{~ms}$, the transmitting particles have moved back to their original position. Figure 3 shows the first $\mathrm{S}$-wave lobe $(-x)$ has reached receiver $R 3$ and a new S-wave lobe in the opposite direction $(+x)$ exists and approaches receiver $R 4$. These two S-wave lobes form a vortex in the zone between receivers $R 5$ and $R 3$. At $30 \mathrm{~ms}$, the first S-wave lobes $(-x)$ have reached the farmost receiver but are greatly attenuated. The second $\mathrm{S}$-wave lobe $(+x)$ has passed receiver $R 3$. And a third S-wave lobe $(-x)$ has been generated and has reached receiver $R 5$. These three S-wave lobes form two vortices as clearly shown in Figure 3 on the $\mathrm{x}$-z plane at $20 \mathrm{~ms}$ and $30 \mathrm{~ms}$ after initiation. Finally, at $40 \mathrm{~ms}$, the first S-wave lobe vanishes. The second and the third S-wave lobes have proceeded to receiver $R 1$ and receiver $R 3$, respectively. No appreciable new S-wave lobe is generated after the third lobe.

On the $y$-z plane, the arrows of velocity vectors are much shorter than those on the $x$-z plane (Figure 3 ), indicating that the main particle motions occur in the direction perpendicular to this plane. However, small disturbances can still be observed near the axis of the specimen. The zone of disturbance progresses as the $\mathrm{S}$-wave propagates through the specimen.

To illustrate the complicated three-dimensional nature of the particle motions, the particle velocity vectors at $30 \mathrm{~ms}$ on five cutting planes going through the five primary receivers, respectively, with their normal parallel to the $z$-axis are shown in Figure 4. The particle motions shown in Figure 4 are consistent with the observations made from Figure 3. Horizontal motions in the negative $x$-direction are seen on plane $x-y-R 1$ (the first S-wave lobe) and plane $x-y-R 5$ (the third S-wave lobe). Stronger positive- $x$ horizontal motions (the second S-wave lobe) occur on plane $x-y-R 3$ and plane $x-y-R 4$. The main shear motions are limited to a central circular zone with a diameter of approximately one-half the diameter of the specimen. Vortices are also observed on plane $x-y-R 5, x-y-R 4$ and $x-y-R 3$. As clearly shown on plane $\mathrm{x}-\mathrm{y}-\mathrm{R} 5$, two vortices are formed symmetrically on each side of the main zone of perturbation.

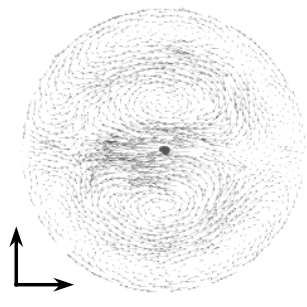

R5

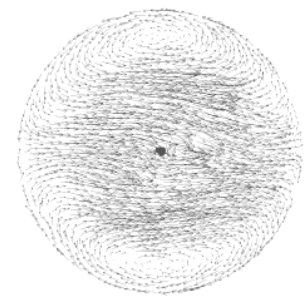

R4

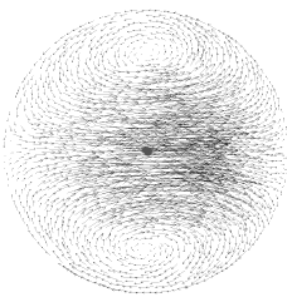

R3

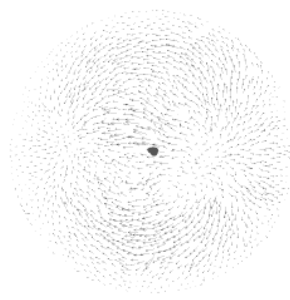

R2

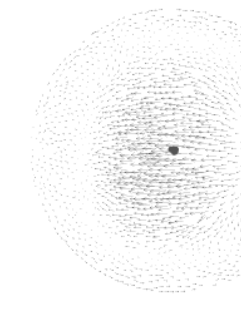

R1

202 Figure 4. Particle velocity vectors on $x-y$ planes at $30 \mathrm{~ms}$, through each receiver. Note that the scale for the velocity vectors is the same as in Figure 3. 
The upward and downward particle motions observed in Figure 3 imply coupled P-S-wave propagation in the specimen. To better study this phenomenon, both shear strain $\left(\varepsilon_{x z}\right)$ and axial strain $\left(\varepsilon_{z z}\right)$ contours on $x$-z-plane at $10 \mathrm{~ms}$ and $20 \mathrm{~ms}$ are shown in Figure 5. Local strain is calculated from volume-averaged measurements of strain rate and the current time step for the simulation. In Figure 5, shear strains are clearly shown in the zones where large horizontal motions occur as indicated in the velocity vectors field. The formation, progression and vanishing of the shear strain localization zones exactly coincide with the shear wave lobes shown above in Figure 3. In Figure 5, a P-wave is also illustrated by the alternating contractive and dilatant local strains, which are antisymmetric with respect to the central axis. Comparing shear strain to axial strain, it is clear that the P-wave propagates faster than the S-wave.

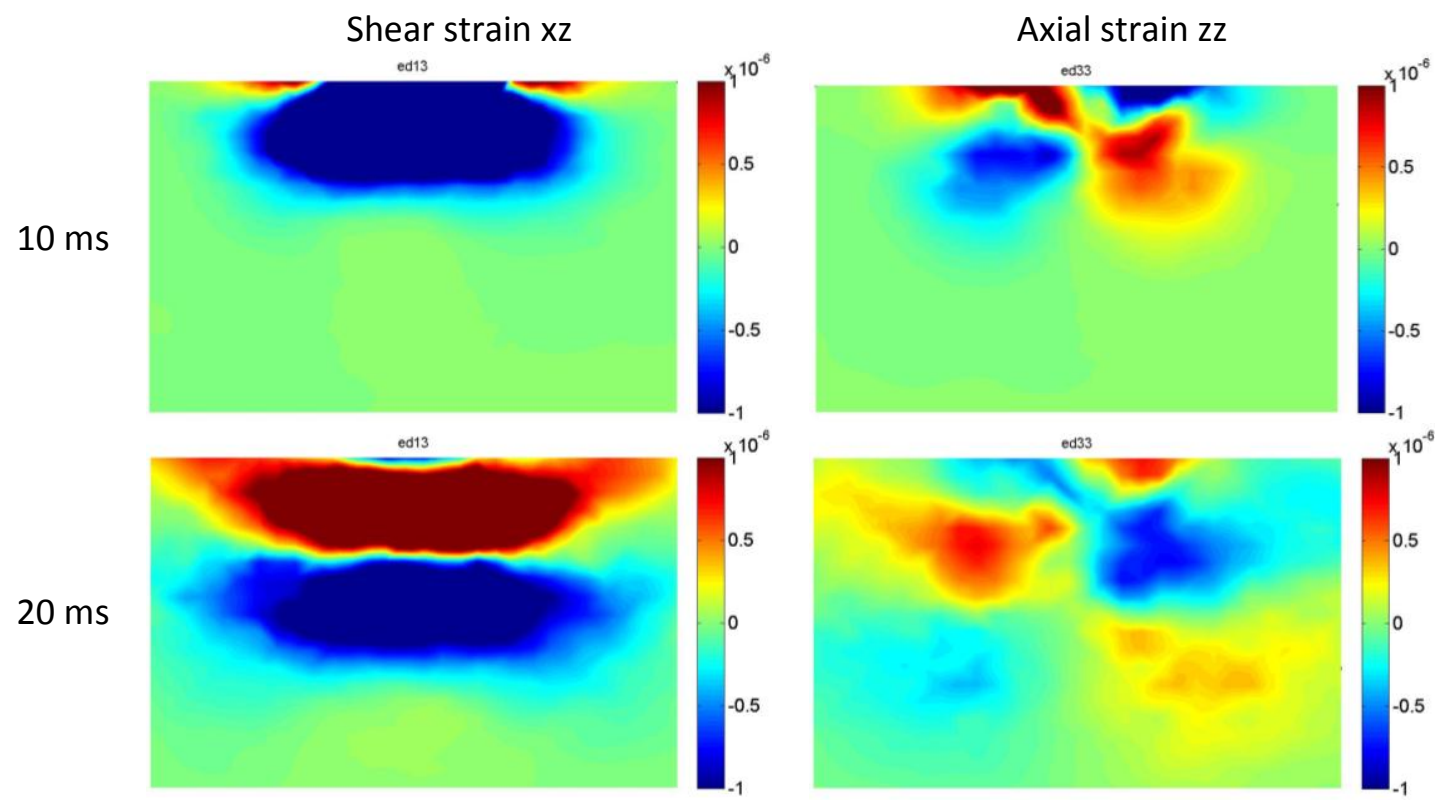

Figure 5. Contours of shear strain-xz and axial strain-zz on xz-plane at $10 \mathrm{~ms}$ and $20 \mathrm{~ms}$.

214 To examine the effects of P-wave interference on S-wave velocity measurements, displacements in the $x$ and $z$-directions of primary receiver $R 3$ and the four complementary receivers $R 6, R 7, R 8$ and $R 9$ are measured and shown in Figure 6 . The five receivers have approximately the same distance to the S-wave transmitting zone. They are symmetric to the central axis and equally spaced (with $R 3$ in the middle).

Figure 6(a) shows that receivers symmetric to the axis have a similar $x$-displacement response, such as $R 6 / R 9$ and $R 7 / R 8$. The larger the distance to the axis, the smaller the amplitude of receiving signal. Receiver R3, which is located at the axis, has the greatest response. In Figure $6(\mathrm{~b}), R 6 / R 9$ and $R 7 / R 8$, have similar but inverse $z$-displacement receiving signals. Receivers $R 6$ and $R 7$ are at the zone showing downward velocity vectors when the first S-wave is approaching (Figure 3), so the initial polarity of receiving signals is negative as shown in Figure $6(\mathrm{~b})$. Similarly, $R 8$ and $R 9$ are at the zone showing upwards velocity vectors when the first S-wave is approaching, thus showing positive initial polarity. In Figure $6(\mathrm{~b})$, the receiver to axis distance has little effect on the amplitude of the receiving signals but affects the arrival time. It should be noted that $R 3$ has nearly zero $z$-displacement response which implies a smaller P-wave inference along the axis. Thus, P-wave inference does exist when a specimen is 
subjected to S-wave excitation, but if the specimen boundaries are sufficiently distant, the zone along the central axis is less affected by $P$-wave inference.

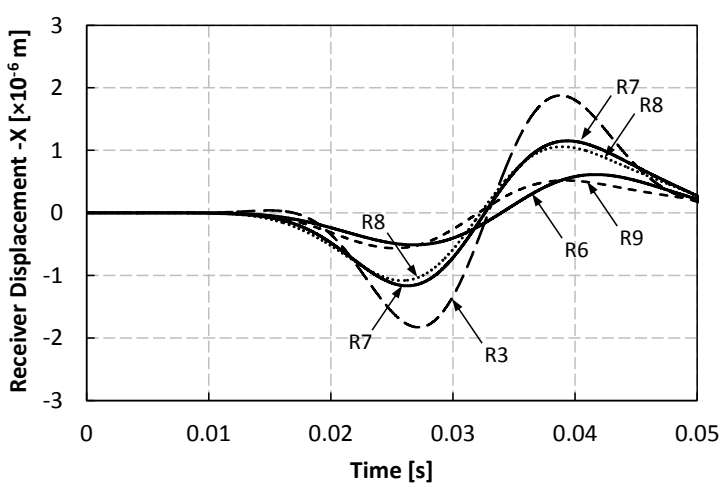

(a)

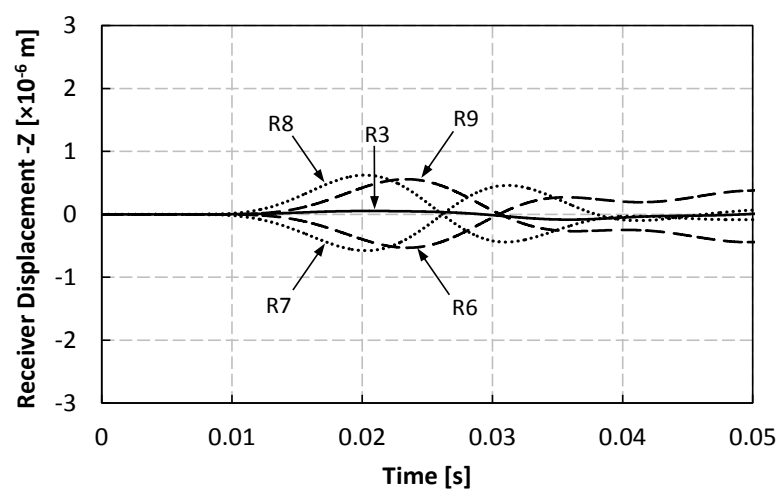

(b)

Figure 6. Receiving signals in terms of: (a) displacement in $\mathrm{x}$ direction; (b) displacement in $\mathrm{z}$ direction.

\section{INTERPRETATION OF RECEIVED SIGNAL}

232 In physical bender element tests, S-wave velocity is calculated from the travel time and the distance between the source and the receiver. The start-to-start and peak-to-peak methods (Viggiani and Atkinson 1995) are often used. In the current simulations, the multiple-receiver configuration makes it possible to calculate S-wave velocity from the travel time and the distance between receivers. Note in Figure 2, though the receiving signals resemble the source signal, there is a higher level of similarity amongst the receiving signals. Key features of the waveform such as the start and peak points are clearly defined in the numerical receiving signals due to their noise-free quality, which to a great extend reduces the uncertainties associated with determining S-wave velocity.

In Figure 5, since the shear strain contours can be determined directly from the DEM simulations, they provide a fundamental approach for determining S-wave velocity as shown in Figure 7. In Figure 7, a square grid is placed on the shear strain contours so the travel distance of the shear wave at different time points can be accurately measured. Then the S-wave velocity can be readily obtained by dividing the travel distance by the time difference. In Figure 7, because the front edge of the first shear lobe (in blue) is not very clearly defined, the front edge of the second shear lobe (in red) was used as a reference. It reached $0.6 \mathrm{~m}$ and $1.5 \mathrm{~m}$ at $15 \mathrm{~ms}$ and $20 \mathrm{~ms}$ respectively after excitation. So the S-wave velocity is: $(1.5 \mathrm{~m}-0.6 \mathrm{~m}) /(20 \mathrm{~ms}-15 \mathrm{~ms})=180 \mathrm{~m} / \mathrm{s}$. This value will be used as a baseline value to examine the validity of other signal-based S-wave determination methods that follow.

Figure 8(a) shows S-wave velocities calculated between the source and each of the five primary receivers and Figure 8 (b) shows S-wave velocities calculated between four pairs of neighboring primary receivers based on both $x$-displacement and $x$-velocity signals. In general, the S-wave velocities calculated by different methods based on both displacement and velocity signals are consistent for receivers $R 1, R 2$, and $R 3$. Due to near field effects (Sanchez-Salinero et al. 1986; Arroyo 2003), the Swave velocities calculated from receivers $R 4$ and $R 5$ are relatively higher than average (which also affects the accuracy of S-wave velocities determined between pairs $R 3-R 4$ and R4-R5). The S-wave 
than those determined using peak-to-peak method as shown in Figure 8(a). This variation is due to the fact that the receivers do not necessarily respond at the same excitation frequency that the source particles do (thus the lags between the start point and the peak point could be different), which is further discussed below. In the following discussion, S-wave velocity will be determined by averaging the results obtained using receiver to receiver analysis from receiver pairs $R 1-R 2$ and $R 2-R 3$ based on the peak to peak method. This averaged S-wave velocity for the case shown in Figure 8 is $180 \mathrm{~m} / \mathrm{s}$. This is consistent with the baseline S-wave velocity determined based on shear strain contours in Figure 7.

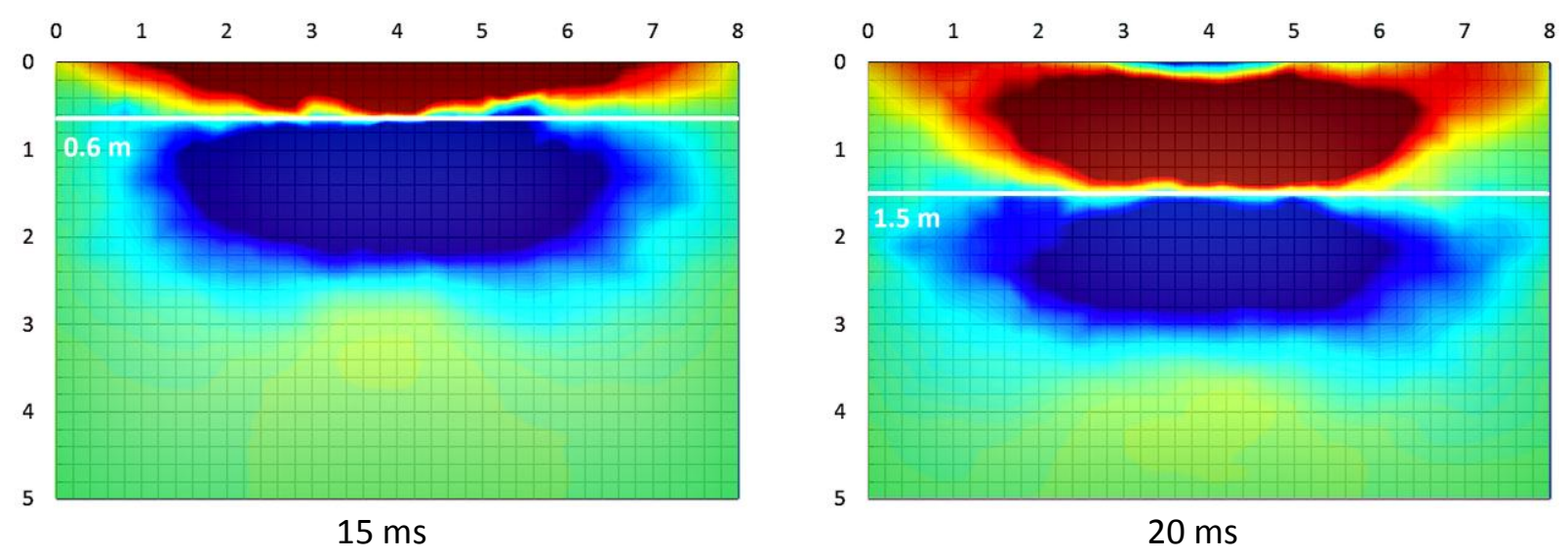

264 Figure 7. Determining travelling distance of shear wave based on graduated shear strain contours.

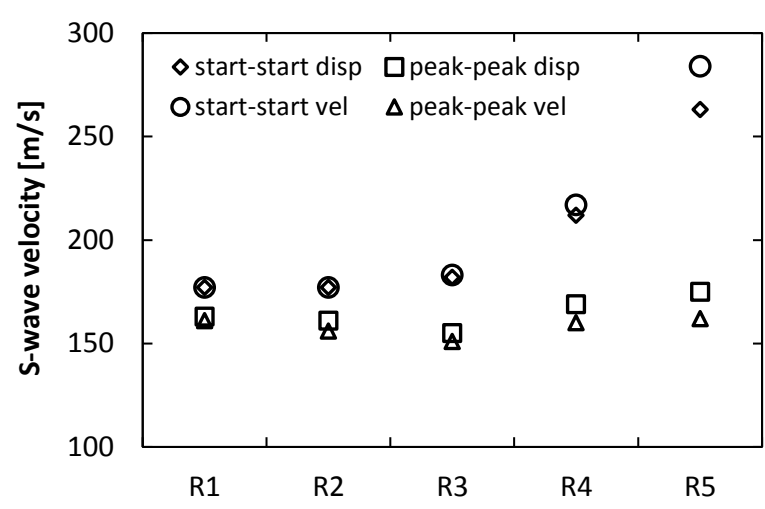

(a)

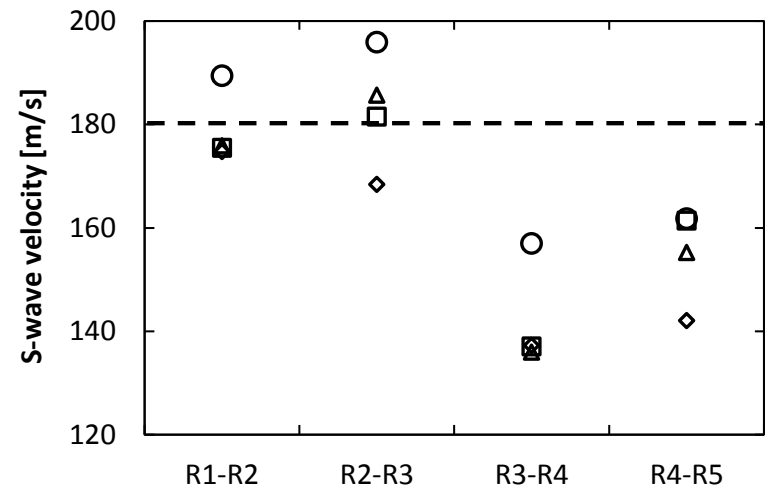

(b)

Figure 8. $V_{s}$ determined by different methods. (a) source to receivers analysis; (b) receiver to receiver analysis

Another commonly used method to determine S-wave velocity in the time domain is cross-correlation (Viggiani and Atkinson 1995), a signal processing operation used to identify similarities between two time-shifted signals (Santamarina and Fratta, 2005). When it is applied to the bender element test, the waveforms of the two signals are required to be highly similar so as to effectively determine the S-wave travel time, yet this is not always well satisfied between a source signal and a receiving signal in a physical test. In DEM simulations, cross-correlation can be readily applied between any two receivers. Cross-correlation is useful in automating the S-wave velocity calculation, especially when continuous measurement is required.

The length of the signal used to perform cross-correlation has a significant effect on the results, which has been seldom discussed in previous studies. To study the effect of signal length, cross-correlation was 
performed on receiver pairs $R 1-R 2$ and $R 2-R 3$ based on both $x$-displacement and $x$-velocity signals with various signal lengths from 0.04-0.10 s. The calculated S-wave velocities based on the travel time determined from cross-correlation are shown in Figure 9. As shown in Figure 9, a significant variation was observed when different signal lengths were used. The cross-correlation method over predicted the $\mathrm{S}$-wave velocity when either the signal length is too short or too long. The largest error is $88 \%$ compared to the baseline value when the signal length is $0.1 \mathrm{~s}$. The calculated S-wave velocities are consistent with the reference value when the signal length is $0.055-0.060 \mathrm{~s}$. This can be explained by noting that $0.055-$ $0.060 \mathrm{~s}$ is approximately the time it takes for the S-wave to reach the bottom boundary of the specimen before reflection occurs. Receiving signals longer than $0.06 \mathrm{~s}$ contain information of the reflecting wave while short receiving signals contains incomplete information; either case can lead to misinterpretation of the S-wave travel time. Therefore, the length of signals should be carefully selected when using crosscorrelation to determine S-wave velocities.

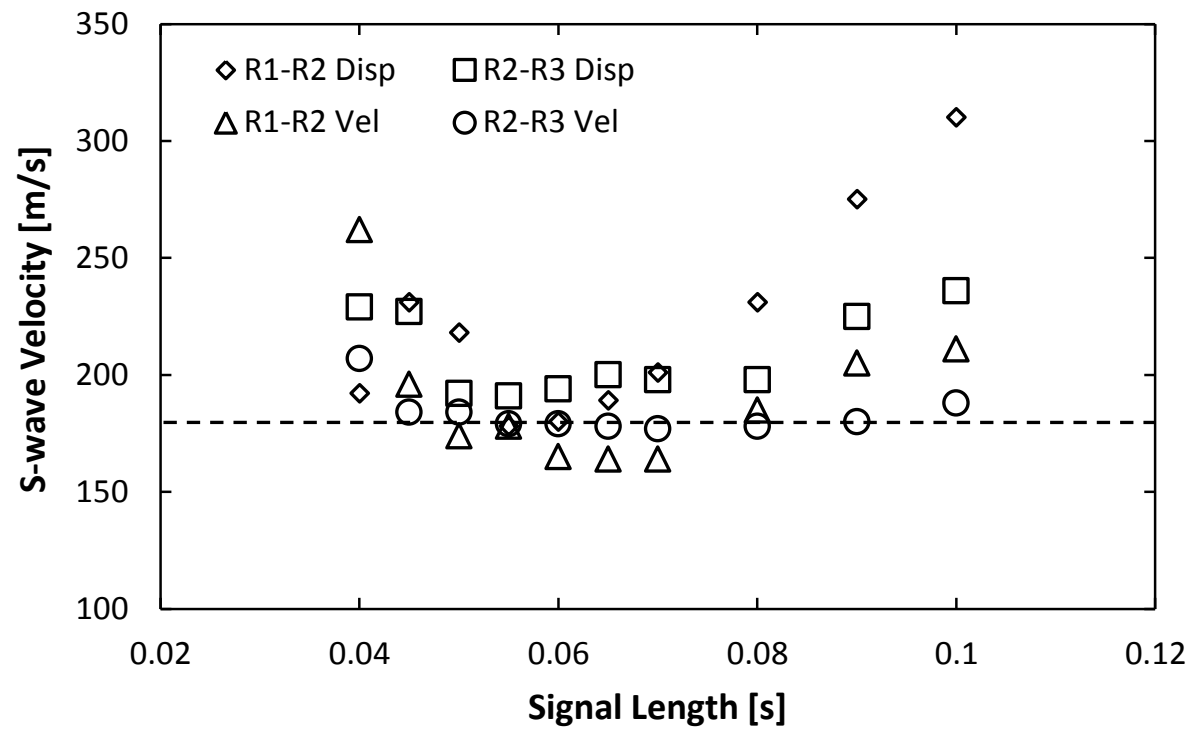

Figure 9. Effect of signal length on S-wave velocity determination by cross-correlation. The average S-wave velocity from start-start and peak-peak measurements is shown as a horizontal dashed line for comparison.

\section{EFFECTS OF EXCITATION SIGNAL}

\section{Effects of excitation amplitude}

As noted above, the shear stiffness of granular material is a function of strain level, thus the S-wave velocity varies as it is measured at different strain levels. In laboratory bender element tests, the shear strain induced in the specimen is controlled by the amplitude of the excitation signal. In the current study, a series of simulations were performed with various excitation amplitudes (amplitude $/ D_{50}=5 \times 10^{-}$ $6 \sim 5 \times 10^{-2}$ ) and the S-wave velocities were determined and are shown in Figure 10. It is seen that S-wave velocity is constant when the amplitude/ $D_{50}$ ratio is low $\left(<1 \times 10^{-4}\right)$ and then decreases as the excitation amplitude is further increased. Similar observations were have been made from bender element test results (Montoya et al 2013). The degradation in inter-particle stiffness and thus, the S-wave velocity, is due to the transition from elastic to plastic behaviors at contact (i.e., contact slip) (Santamarina et al. 2001; Agnolin and Roux 2007). In the current study, contact slip was monitored within the DEM 
specimen as shown in Figure 11. Figure 11 shows the fraction of contacts where slip is occurring during the first $100 \mathrm{~ms}$ of wave propagation $(\Delta \mathrm{t}=1 \mu \mathrm{s})$. Small normalized excitation amplitudes (i.e., $5 \times 10^{-6}$ and $5 \times 10^{-5}$ ) have almost the same slip fraction, which is consistent with the computed shear wave velocity values shown in Figure 10 for these two excitation amplitudes (i.e., they are very similar). The sliding fraction for a normalized amplitude of $5 \times 10^{-4}$ shows a slight increase compared to the smaller normalized amplitudes, which explains the small decrease in shear wave velocity value shown in Figure 10. Further increases of normalized amplitude result in considerable increases in the sliding fraction, which are accompanied by considerable decreases in shear wave velocity, as shown in Figure 10.

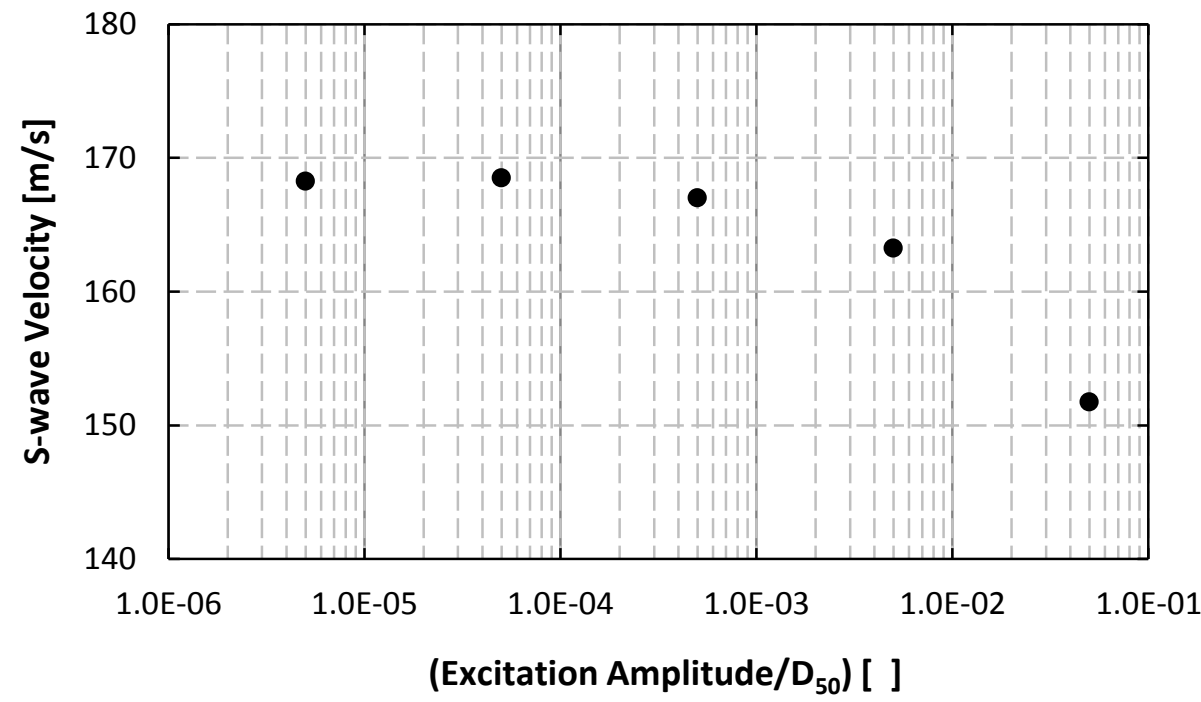

Figure 10. S-wave velocity versus excitation amplitudes

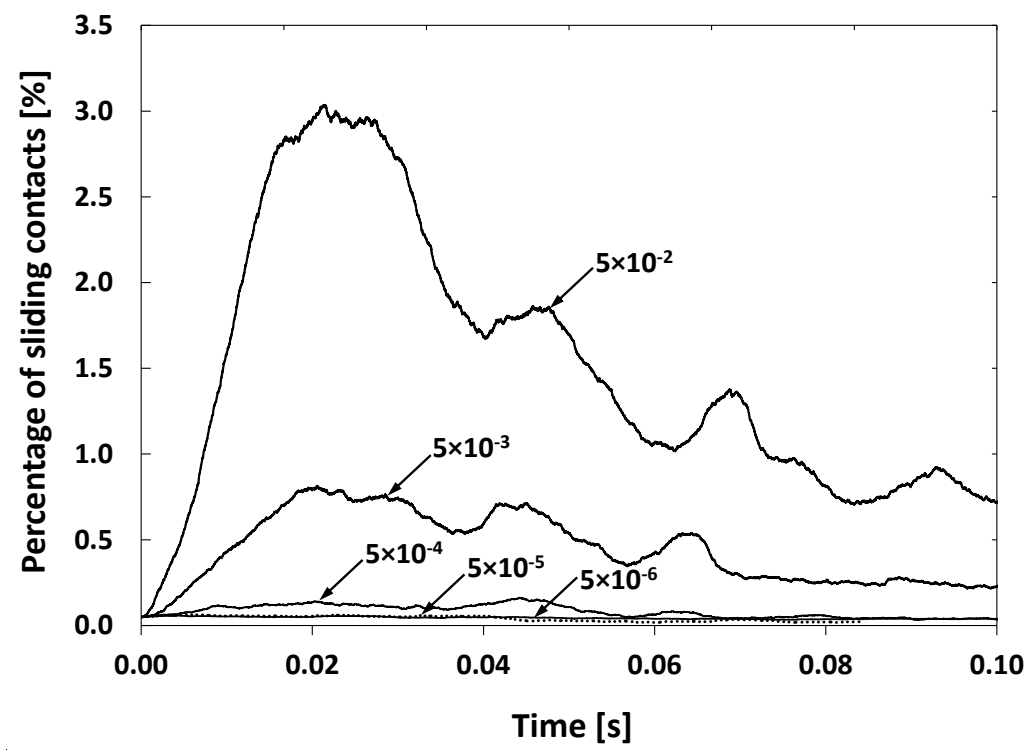

Figure 11. Percentage of sliding contacts for different values of Amplitude $/ d_{50}$ 


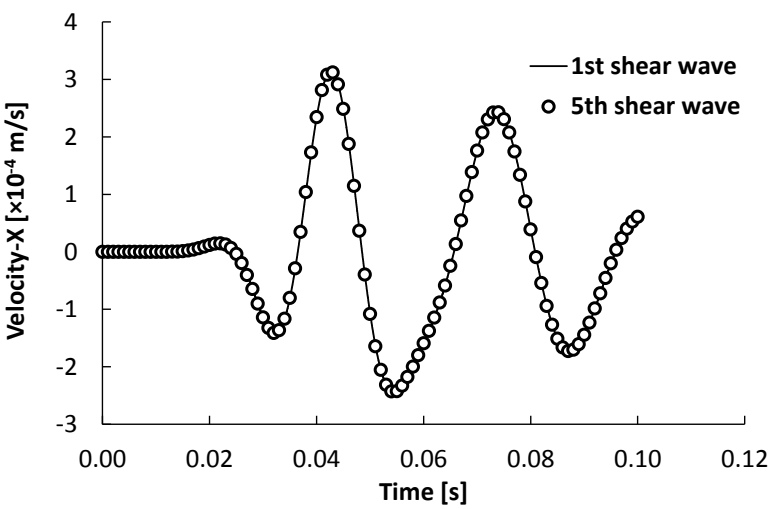

(a) amplitude $/ D_{50}$ ratio less than $1 \times 10^{-4}$. wave, indicating assembly's fabric remains unchanged.

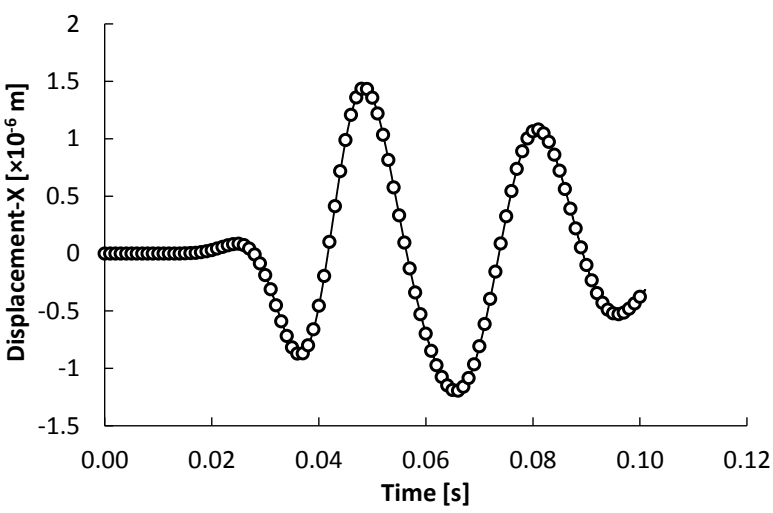

(b)

The previous section shows the importance of excitation amplitude in determining shear wave velocity. Specifically, care should be taken when choosing the excitation amplitude to avoid plastic response at particle contacts. To ensure that the particle assembly is elastic under shear wave excitation, five shear waves with an amplitude/ $D_{50}$ equal to $5 \times 10^{-5}$ were applied to the assembly and time history responses of each receiver for different shear waves were recorded. If the amplitude of the shear stress passing through the soil is large enough to cause rotation or sliding of particles, the soil fabric is altered and different responses will be expected for successive application of shear waves. Figure 12 shows the horizontal velocity and displacement time histories of receiver R1 during application of the first and fifth shear waves. It can be seen that the time histories match each other, indicating of elastic behavior. Therefore, this amplitude is used throughout this study for determination of shear wave velocity. This result is consistent with Figure 10 which shows a constant shear wave velocity for an excitation

To investigate elasticity at the microscale, spherical histograms were used to describe the threedimensional spatial distribution of contact orientation, normal force, and shear force in the particulate assembly as shown in Figure 13. The normalized amplitude of the applied shear wave was $5 \times 10^{-5}$. For contact orientation, the radius of the spherical histogram for a given solid angle $d \Omega$ is the total number of contacts within $d \Omega$ normalized by the total number of contacts in the assembly. For normal and shear force, the radius of the spherical histogram for a given solid angle $d \Omega$ is the ratio of the mean of the contact normal or shear forces having orientations within $d \Omega$ normalized by the mean contact normal force in the assembly. It is observed that all the histograms are the same during the application of shear

Figure 12. Comparison of horizontal (a) velocity and (b) displacement time histories recorded at receiver R1 during application of first and fifth shear waves.

\section{EFFECTS OF PARTICLE SIZE}

The effect of particle size on S-wave velocity has been widely studied by using bender element tests. Recent work by Yang and Gu (2012) found contradictory results when comparing results of previous researchers. DEM simulation allows for the study of particle size effect with a much larger size range 
than physical test does. Knowledge of the particle size effects on wave propagation is important for DEM simulations to assess the validity of mass-scaling to decrease computation time (e.g. Evans and Frost 2007, Jacobson et al. 2007, Belheine et el. 2009).
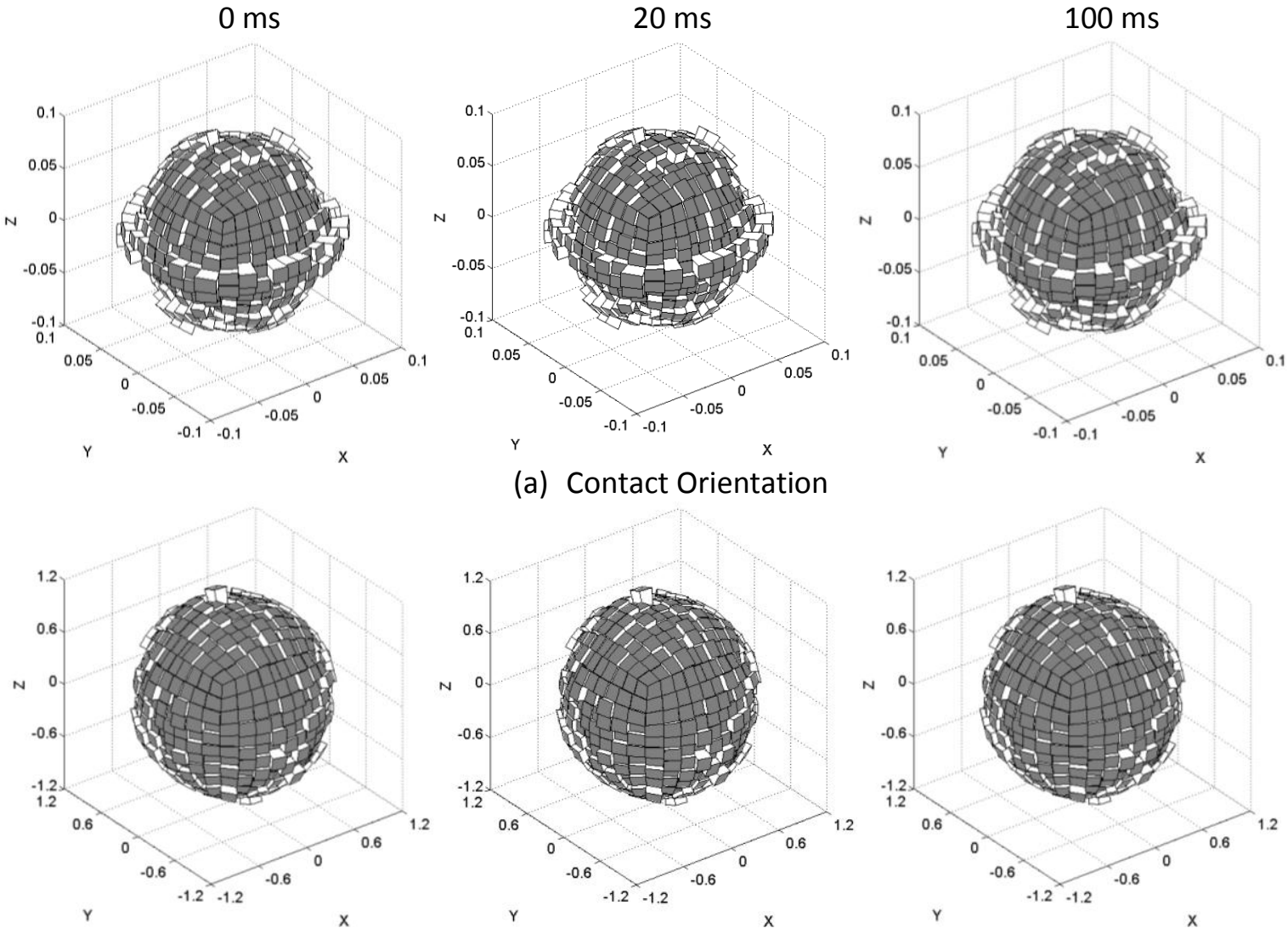

(b) Contact Normal Force
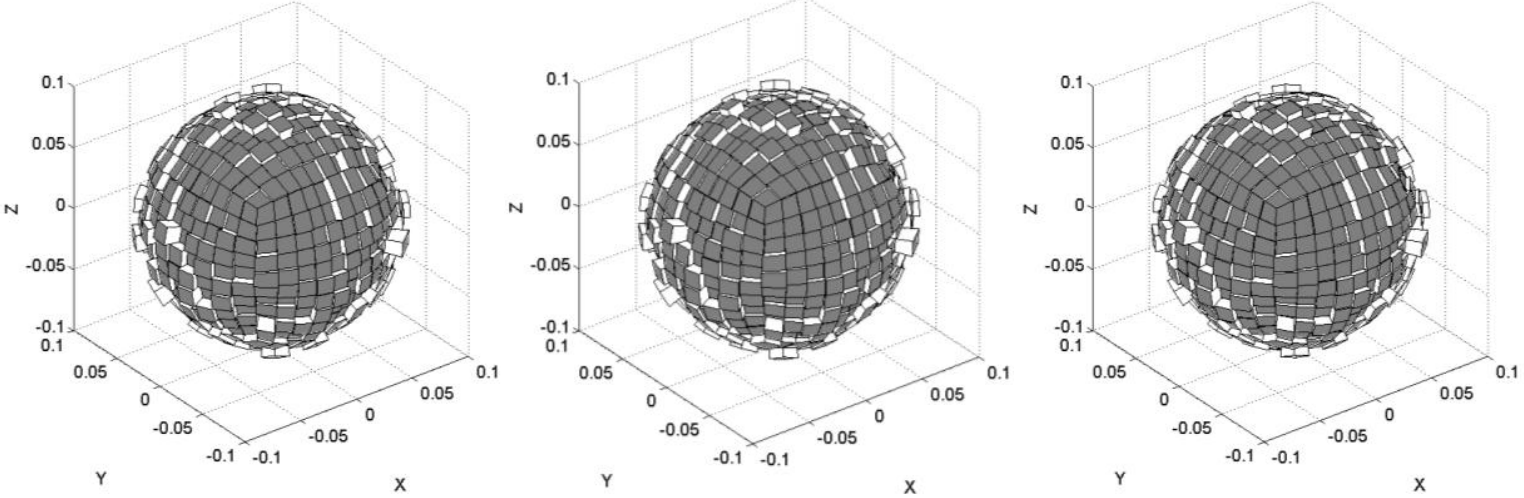

(c) Contact Shear Force

347 Figure 13. Spherical histogram of (a) contacts' orientation; (b) contacts' normal force; (c) contacts' shear force during application of shear wave velocity (Normalized amplitude $=5 \times 10^{-5}$ ).

349 Three mean particle sizes were considered ( $2 \mathrm{~mm}, 20 \mathrm{~mm}$, and $200 \mathrm{~mm}$ ). The number of particles was 350 kept constant and only particle size was changed. Since wave propagation is a dispersive phenomenon 351 involving high frequency effects, different responses are expected from models with different particle sizes. Figure 14 shows the effects of particle size on S-wave velocity and resonant frequency. There was 
little change in S-wave velocity over two orders of magnitude in particle size. This agrees with results presented by Yang and Gu (2012), who found that S-wave velocity is effectively size independent. Regarding the resonant frequency of the model, power law behavior was observed. These results indicate that mass-scaling (which is used in current study) can be applied to reduce computing time in DEM simulations of S-wave propagation without altering the simulated S-wave velocity. The excitation frequency should be carefully selected near the resonant frequency (a function of the particle size) to obtain strong frequency response in the model.

360

361

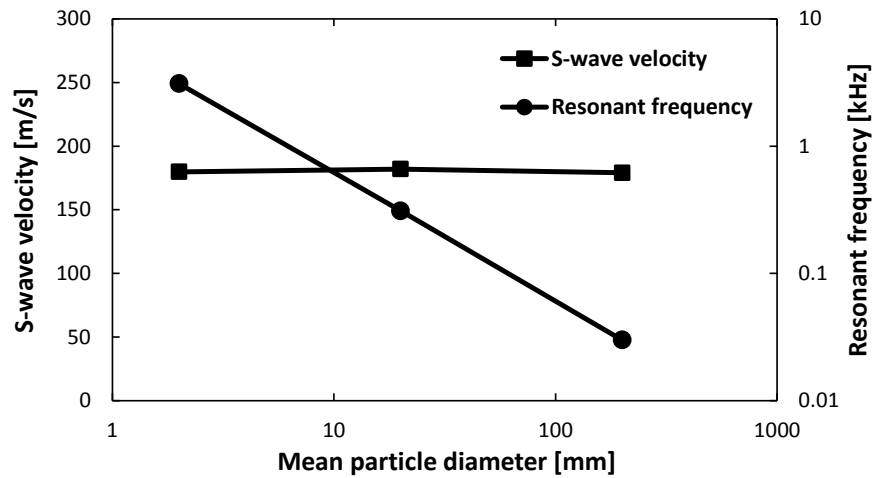

Figure 14. Effects of particle size on S-wave velocity and resonant frequency.

\section{EFFECTS OF STRESS STATE AND PARTICLE MATERIAL PROPERTIES}

Stress state affects interparticle stiffness (e.g., Santamarina et al. 2001) and, perhaps more significantly, contact quality (Evans et al. 2011) and thus, wave propagation speed. Experimental evidence and analytical studies support the choice of a power function to relate velocity and effective mean stress, $p^{\prime}$, which can be expressed as:

$$
V_{s}=\alpha\left(\frac{p^{\prime}}{1 k P a}\right)^{\beta}
$$

where $\alpha$ and $B$ are fitting parameters. $\alpha$ represents the S-wave velocity under $1 \mathrm{kPa}$ effective mean stress and $B$ reflects the sensitivity of the S-wave velocity to the change in effective mean stress. Both $\alpha$ and $B$ vary with the particle material properties, contact behavior and packing. The Hertzian contact model used in the current study is defined by two material properties: particle shear modulus $G_{g}$ and particle Poisson's ratio $v_{g}$. Interparticle secant normal stiffness using the Hertzian contact model is defined as:

$$
k_{n}=\frac{2 G_{g} \sqrt{2 R}}{3\left(1-v_{g}\right)} \sqrt{U}
$$

where $U$ is the overlap of the two particles (the so-called soft-contact approach) and $R$ is the equivalent radius of two particles $(a$ and $b$ ) in contact: 


$$
R=\frac{2 R_{a} R_{b}}{R_{a}+R_{b}}
$$

The interparticle tangent shear stiffness for the Hertzian contact model is defined as:

$$
k_{s}=2 \frac{\sqrt[3]{3 G_{g}^{2}\left(1-v_{g}\right) R}}{2-v_{g}} \sqrt[3]{F_{n}}
$$

where $F_{\mathrm{n}}$ is normal contact force.

381

382

383
In order to study the effect of stress state, particle shear modulus $\left(G_{g}\right)$, particle Poisson's ratio, $\left(v_{g}\right)$, and interparticle friction $(\mu)$ on shear wave velocity $\left(V_{s}\right)$, a series of simulations were performed with different combinations of $G_{g}, v_{g}$, and $\mu$. In each series, S-wave velocities were measured under different effective isotropic confining stress levels ranging from $150 \mathrm{kPa}$ to $1200 \mathrm{kPa}$. The characteristics of each simulation series are shown in Table 2 . In all simulation series, $V_{s}$ increases as effective mean stress increases. The slopes of the $V_{s}$ versus $p^{\prime}$ curves become smaller as effective stress increases, indicating a decreasing effect of increasing stress on $V_{s}$. This trend is similar to that observed in laboratory tests (Santamarina et al. 2001; Montoya et al. 2013).

Table 2 Simulation characteristics

\begin{tabular}{ccccccc}
\hline $\begin{array}{c}\text { Series } \\
\text { No. }\end{array}$ & $\begin{array}{c}G_{g} \\
(\mathrm{GPa})\end{array}$ & $v_{g}$ & $\mu$ & $\begin{array}{c}p^{\prime} \\
(\mathrm{kPa})\end{array}$ & $\begin{array}{c}\alpha \\
(\mathrm{m} / \mathrm{s})\end{array}$ & $\beta$ \\
\hline T-1 (Iso) & 2.9 & 0.31 & 0.31 & $150,300,600,900,1200$ & 79.50 & 0.16 \\
\hline T-2 (Iso) & 6.0 & 0.31 & 0.31 & $150,300,600,900,1200$ & 124.95 & 0.10 \\
\hline T-3 (Iso) & 9.0 & 0.31 & 0.31 & $150,300,600,900,1200$ & 178.01 & 0.05 \\
\hline T-4 (Iso) & 2.9 & 0.26 & 0.31 & $150,300,600,900,1200$ & 67.28 & 0.18 \\
\hline T-5 (Iso) & 2.9 & 0.36 & 0.31 & $150,300,600,900,1200$ & 76.85 & 0.17 \\
\hline T-6 (Iso) & 2.9 & 0.31 & 0.50 & $150,300,600,900,1200$ & 78.60 & 0.16 \\
\hline T-7 (Ko $)$ & 2.9 & 0.31 & 0.31 & $150,300,600,900,1200$ & 66.24 & 0.19 \\
\hline
\end{tabular}

Figure 15(a) shows that higher $G_{g}$ results in higher $V_{s}$ (reflected by higher $\alpha$ ), but lower sensitivity to the change in stress state (reflected by lower $B$ ). Physically, this implies that specimens are more sensitive to changes in particle stiffness at low mean effective stress than at high mean effective stresses. At higher mean stress, softer particles have higher contact radii than stiffer particles. Thus, increasing mean stress leads to improved contact quality (e.g., Yun and Evans 2010) which compensates for the smaller wave velocity through the individual low-stiffness particles, causing measured velocities to be similar at high stresses. This behavior is not entirely surprising when the Hertzian normal contact equation for a simple cubic (SC) packing (Santamarina et al. 2001) is considered:

$$
\frac{r_{c}}{R}=\sqrt[3]{\frac{3\left(1-v_{g}\right)}{2} \frac{\sigma}{G_{g}}}
$$


where $r_{c}=$ contact radius, $\sigma=$ normal stress, and other terms are as previously defined. This expression clearly shows the nonlinear relationship between contact radius and grain stiffness. The implications of this relationship for stiffness values considered in the current work are presented in Figure 16, which shows the more rapid increase in contact radius with increasing stress for the softer particles than the stiffer particles. Note that if contact area were plotted rather than contact radius, the differences would be even more pronounced.

Figure $15(b)$ indicates that decreasing $v_{g}$ results in decreased $V_{s}$ and vice-versa (note that this is also consistent with Equation 6 ). The three $V_{s}$ versus $p$ curves are nearly parallel, indicating similar $B$ values. The effect of interparticle friction, $\mu$, on $V_{s}$ is shown in Figure $15(\mathrm{c})$, which implies that $V_{s}$ is almost the same for $\mu$ values of 0.31 and 0.50 . It can be concluded that $\mu=0.31$ is high enough to avoid sliding in the assembly and therefore $\mu>0.31$ has little effect on $V_{s}$.

The effect of stress state was also investigated. Samples were first isotropically consolidated to a confining pressure of $50 \mathrm{kPa}$ and then deviatoric stress was applied under $K_{0}$ condition until reaching a desired mean effective stress value. Results shown in Figure 15(d) indicate that the shear wave velocity is the same for isotropic and $K_{0}$ states of stress when mean effective stress is the same. This finding confirms the validity of equation (2) and is in agreement with the experimental results of Yanagisawa (1983).

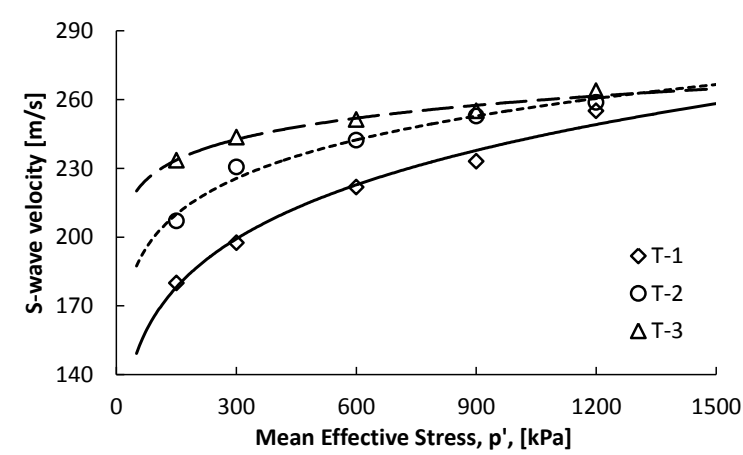

(a)

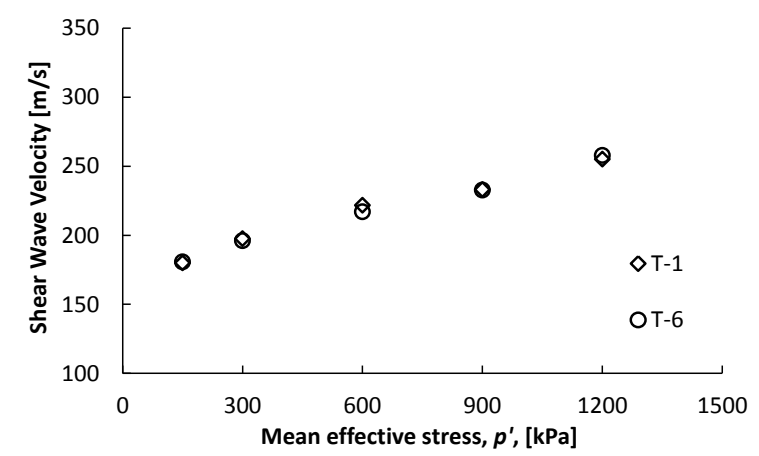

(c)

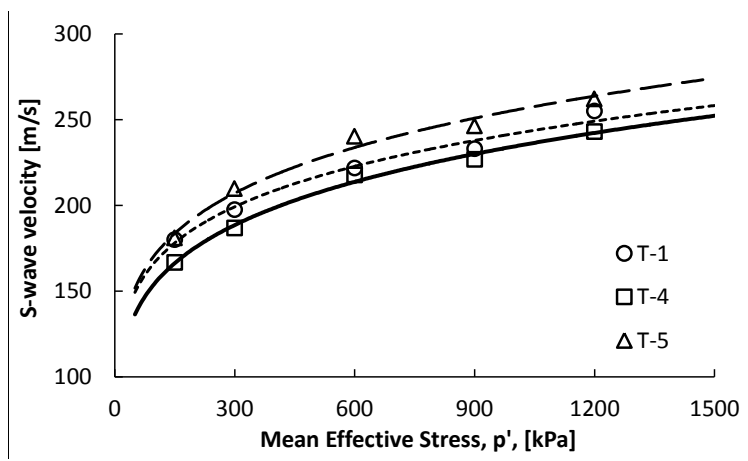

(b)

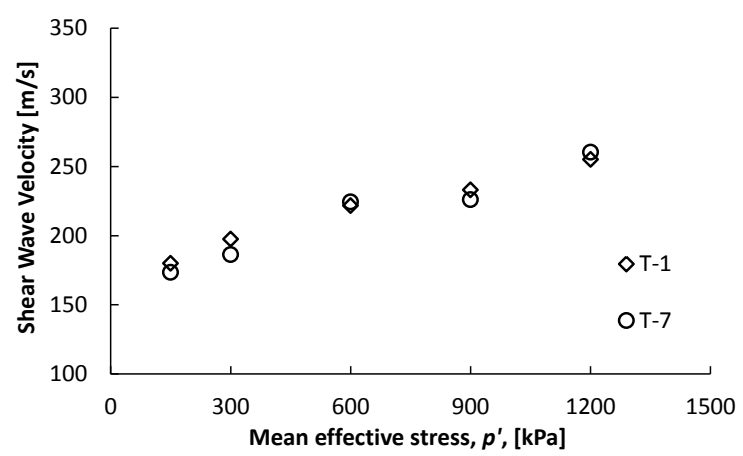

(d)

417 Figure 15. $v_{s}$ versus $p^{\prime}$. (a) effect of particle shear modulus $G_{g}$; (b) effect of particle Poisson's ratio $v_{g}$; (c) effect of 418 interparticle friction angle; (d) effect of state of stress. Lines in (a) and (b) represent best fits of Equation (2) to the 419 data. Fitting parameters are provided in Table 2. 


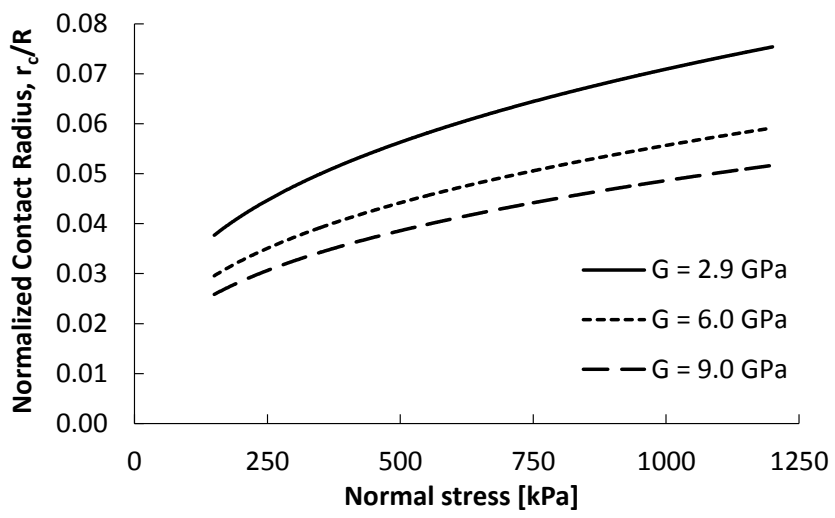

421 Figure 16. Effects of particle stiffness on Hertzian contact radius for a simple cubic (SC) packing.

\section{SUMMARY AND CONCLUSIONS}

423 This paper presents a DEM study of S-wave propagation in random assemblies of spherical particles. 424 DEM simulations provide high quality receiving S-wave signals, given that the responses are free of interfering factors such as cross-talk, system delay, and environmental noise.

The particle motions during wave propagation are illustrated by the evolution of the particle velocity vector field. Vortex-like disturbances are observed ahead of the main shear motions. From the contours of shear strain and axial strain, P-wave interference is observed. However, the zone along the central axis is less affected by P-wave interference. The simulations reveal the complex internal responses of particulate assemblies subjected to S-wave excitation, in aid of a reasonable and correct interpretation of S-wave receiving signals.

432 The multiple-receiver configuration in the present simulation makes it possible to calculate S-wave velocity from the travel time and the distance between receivers. This method produces reliable and relatively precise results when compared to the S-wave velocity measured directly from the shear wave lobes shown on the shear strain contours. The study on the cross-correlation method indicates the length of receiving signal used in cross-correlation method can affect the resulting S-wave velocity, which should be selected appropriately.

S-wave velocity is constant at low excitation amplitude (amplitude/ $D_{50}<10^{-4}$ ) and decreases as the excitation amplitude is further increased. Contact slip is observed when the excitation amplitudes are high which indicates a transition from elastic to plastic inter-particle response.

A wide range of particle sizes are considered. It is found S-wave velocity is effectively size independent while the resonant frequency under the S-wave excitation shows a linearly decreasing trend in log-log space as the particle size increases. These results suggest that mass-scaling can be applied in DEM simulations without altering the resulting S-wave velocity. The excitation frequency should be carefully selected near the resonant frequency (a function of the particle size) in order to obtain strong frequency

446 response in the model. 
The effects of confining stress on S-wave velocity are studied, showing S-wave velocity increases with increasing effective confining stress. The relationship can be described by Eq. (2) which was previously developed based on laboratory tests. Parametric studies on particle shear modulus $G_{g}$ and particle Poisson's ratio $v_{g}$ are performed. It is found that an increase in $G_{g}$ results in higher $\alpha$ but similar $B$ values while the change in $v_{g}$ has minor effects on $\alpha$ and $B$.

\section{ACKNOWLEDGEMENTS}

The second author has received support from the Jerry Yamamuro Fellowship and the Oregon State University School of Civil and Construction Engineering. This support is gratefully acknowledged.

\section{REFERENCES}

Agnolin, I., and Roux, I. (2007). "Internal states of model isotropic granular packings. III. Elastic Properties." Phys. Rev. E, (76), 061304.

Arroyo, M., Muir Wood, D. and Greening, P. D. (2003). "Source near-Field Effects and Pulse Tests in Soils Samples" Géotechnique, 53(3), 337-345.

Belheine N., Plassiard J. P., Donze F. V., Darve F. and Seridi A. (2009). "Numerical simulation of drained triaxial test using 3D discrete element modeling." Computers and Geotechnics, 36(1-2), 320-331.

Blewett, J., Blewett, I. J., and Woodward, P. K. (2000). "Phase and amplitude responses associated with the measurement of shear-wave velocity in sand by bender elements." Can. Geotech. J., 37 13481357.

Burland, J. B. (1989). "Ninth Laurits Bjerrum Memorial Lecture, 'Small is beautiful': The stiffness of soils at small strains." Canadian Geotechnical Journal, 26(4), 499-516.

Clayton, C. R. I. (2011). "Stiffness at small strain: research and practice". Géotechnique, 61(1), 5-37.

Dyvik, R., and Madshus, C. (1985). "Lab Measurements of $G_{\max }$ Using Bender Elements." Advances in the Art of Testing Soils under Cyclic Conditions. Proceedings of a session held in conjunction with the ASCE Convention. ASCE, Detroit, MI, Engl, 186-196.

Evans, T. M., and Frost, J. D. (2007). "Shear banding and microstructure evolution in 2D numerical experiments." Geo-Denver 2007: New Peaks in Geotechnics, February 18, 2007 - February 21, American Society of Civil Engineers, Denver, CO, United states, 28.

Evans, T. M., Lee, J., Yun, T. S., and Valdes J. R. (2011). "Thermal Conductivity in Granular Mixtures: Experimental and Numerical Studies." Deformation Characteristics of Geomaterials, 815-820.

Evans, T. M. and Valdes J. R. (2011). "The microstructure of particulate mixtures in one -dimensional compression: numerical studies." Granular Matter, 13, 657-669.

Fam, M., and Santamarina, J. C. (1997). "A study of consolidation using mechanical and electromagnetic waves." Géotechnique, 47(2), 203-219. 
Fernandez, A., and Santamarina, J. C. (2001). "The Effect of Cementation on the Small Strain Parameters of Sands." Canadian Geotechnical Journal, 38(1), 191-199.

Hardin, B.O., and Richart Jr., F.E. (1963). "Elastic wave velocities in granular soils." Journal of the Soil Mechanics and Foundations Division, 89 33-65.

Itasca. (2009). "PFC 3 : Particle Flow Code in 3 Dimensions." v4.0, Minneapolis, MN.

Jacobson, D. E., Valdes, J. R., and Evans, T. M. (2007). "A numerical view into direct shear specimen size effects." Geotech Test J, 30(6), 512-516.

Lee, J., and Santamarina, J. C. (2005). "Bender elements: Performance and signal interpretation." J. Geotech. Geoenviron. Eng., 131(9), 1063-1070.

Li, L., and Holt, R. M. (2002). "Particle scale reservoir mechanics." Oil and Gas Science and Technology, 57(5), 525-538.

Montoya, B. M., DeJong, J. T., and Boulanger, R. W. (2013). "Dynamic response of liquefiable sand improved by microbial-induced calcite precipitation." Géotechnique, 63(4), 302-312.

Montoya, B. M., Gerhard, R., DeJong, J., Weil, M., Martinez, B., and Pederson, L. (2012). "Fabrication, Operation, and Health Monitoring of Bender Elements for Aggressive Environments." Geotechnical Testing Journal, 35(5), 1-15.

Ning, Z. and T.M. Evans. (2013). "Discrete Element Method Study of Shear Wave Propagation in Granular Soil." Proceedings of the $18^{\text {th }}$ International Conference on Soil Mechanics and Geotechnical Engineering, Paris, France, pp. 1031-1034.

O'Sullivan, C. (2011). Particulate discrete element modelling: a geomechanics perspective. Taylor \& Francis, London.

O'Donovan, J., O'Sullivan, C., and Marketos, G. (2012). "Two-dimensional discrete element modelling of bender element tests on an idealised granular material." Granular Matter, 14, 733-747.

Ouadfel, H. and Rothenburg, L. (2001). "Stress-force-fabric relationship for assemblies of ellipsoids." Mech. Mater. 33, 201-221.

Patel, A., Bartake, P. P., and Singh, D. N. (2009). "An empirical relationship for determining shear wave velocity in granular materials accounting for grain morphology." Geotech Test J, 32(1), 1-10.

Rechenmacher, A.L. (2006). "Grain-scale processes governing shear band initiation and evolution in sands," Journal of the Mechanics and Physics of Solids, 54, 22-45.

Robertson, P. K., Sasitharan, S., Cunning, J. C., and Sego, D. C. (1995). "Shear-wave velocity to evaluate in-situ state of Ottawa sand." J. Geotech. Eng., 121(3), 262-273.

Sadd, M. H., Tai, Q., and Shukla, A. (1993). "Contact law effects on wave propagation in particulate materials using distinct element modeling." Int. J. Non-Linear Mech., 28(2), 251-65.

Sanchez-Salinero, I., Roesset, J. M., and Stokoe, K. H. (1986). "Analytical studies of body wave propagation and attenuation." Rep. No. Report GR 86-15, University of Texas, Austin.

Santamarina, J.C., in collaboration with Klein, K. and Fam, M. (2001). Soils and Waves, J. Wiley and Sons, Chichester, UK, 488 pages. 
Santamarina, J.C. and Fratta, D. (2005), "Discrete Signals and Inverse Problems An Introduction for Engineers and Scientists". J. Wiley and Sons, Chichester, UK, 350 pages.

Saxena, S. K., Avramidis, A. S., and Reddy, K. R. (1988). "Dynamic moduli and damping ratios for cemented sands at low strains." Can. Geotech. J., 25(2), 353-368.

522 Sharma, R., Baxter, C., and Jander, M. (2011). "Relationship between shear wave velocity and stresses at failure for weakly cemented sands during drained triaxial compression." Soils and Foundations, 51(4), 761-771.

Shirley, D. J., and Hampton, L. D. (1978). "Shear-Wave Measurements in Laboratory Sediments." J. Acoust. Soc. Am., 63(2), 607-613.

Styler, M. A., and Howie, J. A. (2012). "Comparing frequency and time domain interpretations of bender element shear wave velocities." GeoCongress 2012, ASCE, Oakland, California, 2207-2216.

Tordesillas, A., Muthuswamy, M., and Walsh, S. (2008). "Mesoscale Measures of Nonaffine Deformation in Dense Granular Assemblies." J. Eng. Mech., 134(12), 1095-1113.

Viggiani, G., and Atkinson, J. H. (1995). "Interpretation of bender element tests." Géotechnique, 45(1), $532 \quad 149-154$.

Woodcock, N.H. (1977). "Specification of fabric shapes using an eigenvalue method". Geol. Soc. Amer. Bull. 88, 1231-1236.

Yanagisawa, E. (1983). "Influence of void ratio and stress condition on the dynamic shear modulus of granular media". in J. T. Jenkins and M. Satake (eds), Advances in the Mechanics and Flow of Granular Materials, Elsevier, Amsterdam, 947-960.

Yang, J., and Gu, X. Q. (2012). "Shear stiffness of granular material at small strains: does it depend on grain size." Géotechnique, 63(2), 165-179.

Yun, T. S., and Evans, T. M. (2010). "Three-dimensional random network model for thermal conductivity in particulate materials." Computers and Geotechnics, 37(7-8), 991-998.

Zhao, X., and Evans, T. M. (2009). "Discrete simulations of laboratory loading conditions." International Journal of Geomechanics, 9(4), 169-178. 